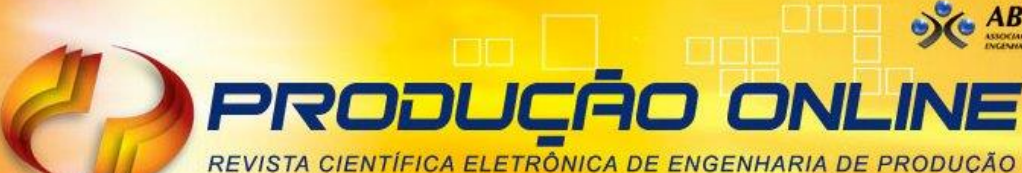 REVISTA CIENTIFICA ELETRÓNICA DE ENGENHARIA DE PRODUÇÃO ISSN 1676-1901
}

\section{PRIORIZAÇÃO DAS ATIVIDADES DE PROJETO NA CONSTRUÇÃO CIVIL ATRAVÉS DA ABORDAGEM MULTICRITÉRIO}

\section{PRIORITIZATION OF PROJECT ACTIVITIES IN CIVIL CONSTRUCTION THROUGH MULTICRITERIA APPROACH}

\author{
Danielle Freitas Santos* E-mail: daniellefreitas.ep@gmail.com \\ Emerson Santos Aguiar** E-mail: emerson.santosaguiar@gmail.com \\ *Universidade Federal de Pernambuco (UFPE), Recife, PE \\ **Universidade Federal de São Paulo (UNIFESP) / Instituto Tecnológico de Aeronáutica (ITA), São \\ Paulo, SP
}

Resumo: O setor da construção civil, apesar da crise, ainda emerge como um segmento relevante para a economia brasileira. Por envolver um nível de complexidade considerável em termos de gestão, planejamento e execução, o setor necessita de alternativas que otimizem a gestão dos recursos, e nesse sentido a abordagem multicritério pode atuar como uma ferramenta eficiente na tomada de decisão na construção civil. A partir disso, o presente estudo objetiva determinar quais grupos de atividades de projeto de uma empresa da construção civil devem ser priorizadas no processo de tomada de decisão. Para tanto, foi utilizado o método multicritério Analytic Hierarchy Process - AHP. Em relação aos resultados obtidos, verificou-se que o critério custo admite relevância significativa na condução do planejamento e controle das ações relacionadas aos projetos, bem como o grupo de atividades "execução de obra" consiste no segmento de atividades mais importante no gerenciamento de projetos da empresa, pelo fato de envolver uma gama de atividades complexas e fundamentais a operacionalização de uma organização da construção civil.

Palavras-chave: Abordagem multicritério. Construção civil. Priorização de atividades de projeto. Analytic Hierarchy Process - AHP.

Abstract: The construction sector, despite the crisis, still emerges as a relevant segment for the Brazilian economy. Because it involves a considerable level of complexity in terms of management, planning and execution, the sector needs alternatives that optimize the management of resources, and in this sense the multicriteria approach can act as an efficient tool in decision making in civil construction. From this, the present study aims to determine which groups of project activities of a construction company should be prioritized in the decision making process. For that, the multicriteria Analytic Hierarchy Process - AHP was used. In relation to the results obtained, it was verified that the cost criterion admits significant relevance in the conduct of planning and control of actions related to the projects, as well as the group of activities "execution of work" consists of the most important segment of activities in the management of projects of the company, because it involves a range of complex and fundamental activities to operationalize a construction organization.

Keywords: Multicriteria approach. Construction. Project activities prioritization. Analytic Hierarchy Process - AHP.

\section{INTRODUÇÃO}

O segmento da construção civil ainda constitui um elo forte da economia nacional, seja através da geração de renda, criação de novos postos de trabalho, entre 
outros aspectos relevantes. A construção civil admite uma cadeia produtiva ampla e complexa, derivada do uso intensivo de tecnologia, da extensa dispersão territorial brasileira e da exigência de qualificação profissional constante.

Compreende todas as áreas e atividades relacionadas a produção de obras, as quais incluem: o planejamento e projeto; execução, manutenção e restauração de obras distintas como estradas, aeroportos, residências, edificações, túneis, saneamento, portos, instalações prediais, entre outros.

Apesar do crescimento constante do setor nas últimas décadas, o relatório da Sondagem da Indústria da Construção - CNI (2017) previu quedas no nível de Utilização da Capacidade de Operação (UCO), que corresponde a porcentagem de uso de toda a capacidade produtiva das indústrias construtoras. O desempenho constante no último relatório (outubro de 2017) indica um percentual de 59\%, abaixo do valor relativo ao mesmo período dos anos de 2016 (60\%).

O nível de atividade da indústria da construção ainda continua em queda (CNI, 2017), porém a índices menos intensos, chegando ao patamar de 46,9. Apesar disso, o segmento ainda opera abaixo do nível ideal de atividade (níveis acima de 50 são considerados crescimento do nível de atividade) e admite alto índice de ociosidade. A esta perspectiva, acrescenta-se o fato do setor ter apresentado uma queda de $6,6 \%$ em relação ao PIB nacional do ano de 2017 (SINICON, 2017).

A partir deste cenário instável, muitas empresas do setor precisam estar atentas as suas atividades, promovendo uma gestão mais eficaz de seus recursos. É neste escopo que a efetivação de um processo de tomada de decisão coerente e fundamentado pode trazer benefícios para a gestão organizacional como um todo, favorecendo uma melhor alocação dos recursos, um planejamento mais eficiente, a redução dos custos e o incremento dos resultados operacionais.

Além disso, o ambiente da construção civil ainda exige um processo de tomada de decisão eficiente e confiável, que precisa ser baseado em dados e informações precisas, bem como na experiência de seus gestores que compreendem a complexidade inerente ao setor. Dessa forma, uma metodologia viável na resolução de problemas ligados a tomada de decisão consiste na abordagem multicritério de apoio a decisão que envolve questões de grande complexidade.

Segundo Gomes et al. (2002), a análise de decisão multicritério compreende ao conjunto de técnicas que visam auxiliar um agente de decisão, seja este um 
indivíduo, grupo de pessoas ou gestores organizacionais, a tomar decisões acerca de um problema complexo, ponderando e escolhendo alternativas viáveis para solucionálo segundo diferentes critérios e pontos de vista.

De acordo com Mota e Almeida (2007), existem atividades em projetos que precisam ser priorizadas em detrimento a sua criticidade e aos fatores envolvidos como custo, variabilidade, influência do clima, uso e mobilização de recursos, nível de qualificação do pessoal envolvido, segurança e outros aspectos, e nesse sentido a aplicação de uma metodologia multicritério pode favorecer a tomada de decisão em relação a projetos.

Dessa forma, tendo em vista a dimensão e complexidade do segmento da construção civil, bem como a relevância de um processo de tomada de decisão coerente nesse âmbito, o presente estudo delimita-se a identificar como a abordagem multicritério pode auxiliar na priorização das atividades de um projeto no escopo da construção civil e determinar quais grupos de atividades de projeto admitem maior relevância na tomada de decisão.

Para tanto, a pesquisa pretende viabilizar uma síntese por meio da aplicação do método Analytic Hierarchy Process (AHP) sobre quais grupos de atividades de projeto devem ser priorizadas no planejamento operacional do setor de Engenharia de uma empresa do segmento da construção civil da cidade de Campina Grande PB, o qual é responsável pelo gerenciamento e decisões relativas aos projetos.

\section{REVISÃO TEÓRICA}

\subsection{Abordagem Multicritério de Apoio à Decisão}

O âmbito organizacional exige constantemente o processo de tomada de decisão por parte dos gestores e em certos casos por parte dos colaboradores em conjunto com a alta cúpula. Geralmente, esse processo envolve fatores como riscos e incertezas, mudanças tecnológicas e de mercado, uma quantidade significativa de partes interessadas envolvidas, entre outros aspectos.

Bana e Costa (1988) afirma que a tomada de decisão é um processo complexo e controverso, onde não cabe apenas decidir entre possíveis alternativas de ações, mas entre distintos pontos de vista e metodologias de avaliação dessas ações, dessa 
forma, é de extrema relevância a consideração de uma múltipla gama de aspectos que possam afetar substancialmente a tomada de decisão.

Nesse contexto, a análise de decisão multicritério consiste em uma metodologia importante no auxílio ao processo de tomada de decisão. De acordo com Almeida (2013), um problema que envolve a decisão multicritério consiste em uma situação onde pelo menos duas alternativas de ação são previstas, e a escolha de qual seja a mais adequada é dirigida pelo desejo de se atender a uma diversidade de critérios distintos, na maioria dos casos conflitantes entre si.

De acordo com Cavalcante e Almeida (2005), a análise de decisão multicritério consiste em uma metodologia de avaliação da decisão que objetiva tornar claro o problema em foco, onde as alternativas são analisadas com base em múltiplos critérios, que geralmente são conflitantes e complexos. Auxilia a decisão em um sistema amplo de alternativas, onde os critérios definidos como relevantes precisam ser considerados simultaneamente, para que a decisão seja fundamentada em aspectos concretos que viabilizem a tomada de decisão eficaz.

Segundo Gomes (2004), a análise de decisão multicritério é um processo que pode ser interpretado como a sequência das seguintes etapas: (i) identificação dos decisores; (ii) definição das alternativas; (iii) definição dos critérios relevantes para o problema de decisão; (iv) avaliação das alternativas em relação aos critérios; (v) determinação da importância relativa dos critérios; (vi) realização da avaliação de cada alternativa; (vii) avaliação global do conjunto das alternativas; e (viii) elaboração das recomendações a serem seguidas.

Os métodos multicritério podem admitir, ainda, várias classificações. Segundo Almeida (2013) tais métodos se classificam em: compensatórios, quando a desvantagem em algum critério pode ser compensada pela vantagem em outro (tradeoff), ou seja, quando os pesos dos critérios representarem uma taxa de substituição e não apenas a ordem de importância relativa dos critérios; e não compensatórios, quando os pesos dos critérios representarem apenas a ordem de importância relativa dos critérios ao invés de representarem um trade-off.

Roy (1996) divide os métodos multicritério em três abordagens principais: critério único de síntese, que visa agregar diferentes visões de uma única função de síntese que pode ser otimizada a posteriori, ou seja, foca as razões de agregação e construção do modelo; sobreclassificação, que considera a criação de uma relação 
de sobreclassificação que indica as preferências do decisor, além de efetuar uma avaliação abrangente desta relação como forma de apoiar a resolução do problema; e métodos interativos, que são métodos que usam a abordagem de tentativa e erro e estruturas de programação matemática multiobjetivo.

Em relação ao contexto da construção civil observa-se na literatura alguns trabalhos envolvendo a aplicação de métodos multicritério de apoio à decisão. Szajubok et al. (2006) propõem um modelo estruturado para o gerenciamento de materiais na construção civil baseado no método multicritério ELECTRE TRI. Mota et al. (2009) apresentam um modelo multicritério para auxiliar os gestores na priorização de atividades de projeto. Famá et al. (2015) estabelecem o caminho crítico de um canteiro de obras mediante o uso da abordagem multicritério associada a técnica PERT/CPM.

Azevedo et al. (2013) utilizam a abordagem multicritério em relação as decisões de orçamentação na construção civil. Lima et al. (2008) empregam a abordagem multicritério em conjunto com um sistema de informação geográfica para determinar a priorização de pavimentos urbanos. Silva et al. (2013) viabilizam uma proposta para identificação de potenciais locais para implantação de uma usina de reciclagem de resíduos da constrição civil mediante a abordagem multicritério.

Há ainda trabalhos envolvendo o uso da abordagem multicritério para a classificação de estoques na construção civil (SZAJUBOK, MOTA e ALMEIDA, 2006); a especificação de um modelo multicritério para a seleção de contratados em projetos de construção civil (ARAÚJO ET AL., 2015); e aplicação da abordagem multicritério na seleção de fornecedores na indústria da construção civil (ALENCAR ET AL., 2007; SCHRAMM ET AL.,2009).

\subsection{Analytic Hierarchy Process - AHP}

O método Analytic Hierarchy Process (AHP) consiste em uma sistemática de apoio à tomada de decisão envolvendo múltiplos critérios. Desenvolvida por Thomas L. Saaty na década de 70, o AHP objetiva dividir o problema em níveis hierárquicos de modo a facilitar sua análise e compreensão. Almeida (2013) indica que o método AHP consiste em uma metodologia de agregação aditiva que foca um procedimento próprio de modelagem das preferências do decisor. 
Ben (2006) conceitua o método AHP como sendo uma metodologia de análise multicritério com base em um processo de ponderação ativa, onde os vários atributos de relevância são representados por meio de sua importância relativa. Toma e Asharif (2003) avaliam que o AHP fundamenta-se na capacidade humana de manipular as informações e na experiência como forma de dimensionar magnitudes relativas por intermédio de comparações par a par.

De acordo com Costa et al. (2008) o modelo AHP admite os seguintes elementos em sua análise: critérios e alternativas, onde uma série de alternativas será comparada em relação a uma série de critérios; correlação binária, onde são realizadas comparações binárias visando verificar a preferência ou indiferença de um elemento em relação a outro; hierarquia, na medida em que os critérios e alternativas são ordenados de forma homogênea baseados na preferência do decisor; e escala fundamental, onde os elementos serão associados a uma escala numérica que evidencia a prioridade de um sobre o outro. Dessa forma a estrutura de aplicação do método AHP segue basicamente as seguintes etapas:

1. Definição do(s) objetivo(s) ou meta(s);

2. Definição dos critérios e alternativas;

3. Estruturação de uma hierarquia;

4. Comparação par a par dos critérios;

5. Criação da matriz de comparação paritária;

6. Verificação da consistência da matriz de comparação;

7. Comparação par a par das alternativas em relação aos critérios;

8. Determinação das matrizes de comparação paritárias das alternativas;

9. Verificação da avaliação global de cada alternativa.

O AHP utiliza de uma abordagem hierárquica para estabelecer critérios e identificar as alternativas (ALMEIDA, 2013). Ou seja, estabelece uma hierarquia para os critérios e alternativas, de forma a categorizar alguma relação de prioridade ou relevância entre estes. Visa decompor um problema em uma estrutura hierárquica descendente que se assemelha a uma árvore genealógica (SAATY, 1980; SCHMOLDT e PETERSON, 1997; GOMES e MOREIRA, 1998).

Além disso, a viabilização de uma estrutura hierárquica facilita a compreensão e análise do modelo, e ainda segundo Bornia e Wernke (2001) a hierarquização possibilita a visualização do modelo como um sistema, evidenciando as interações 
entre elementos e o impacto que estes exercem sobre o sistema. A Figura 1 apresenta o modelo de estrutura hierárquica utilizada no método AHP.

Figura 1 - Modelo de Estrutura Hierárquica do Método AHP

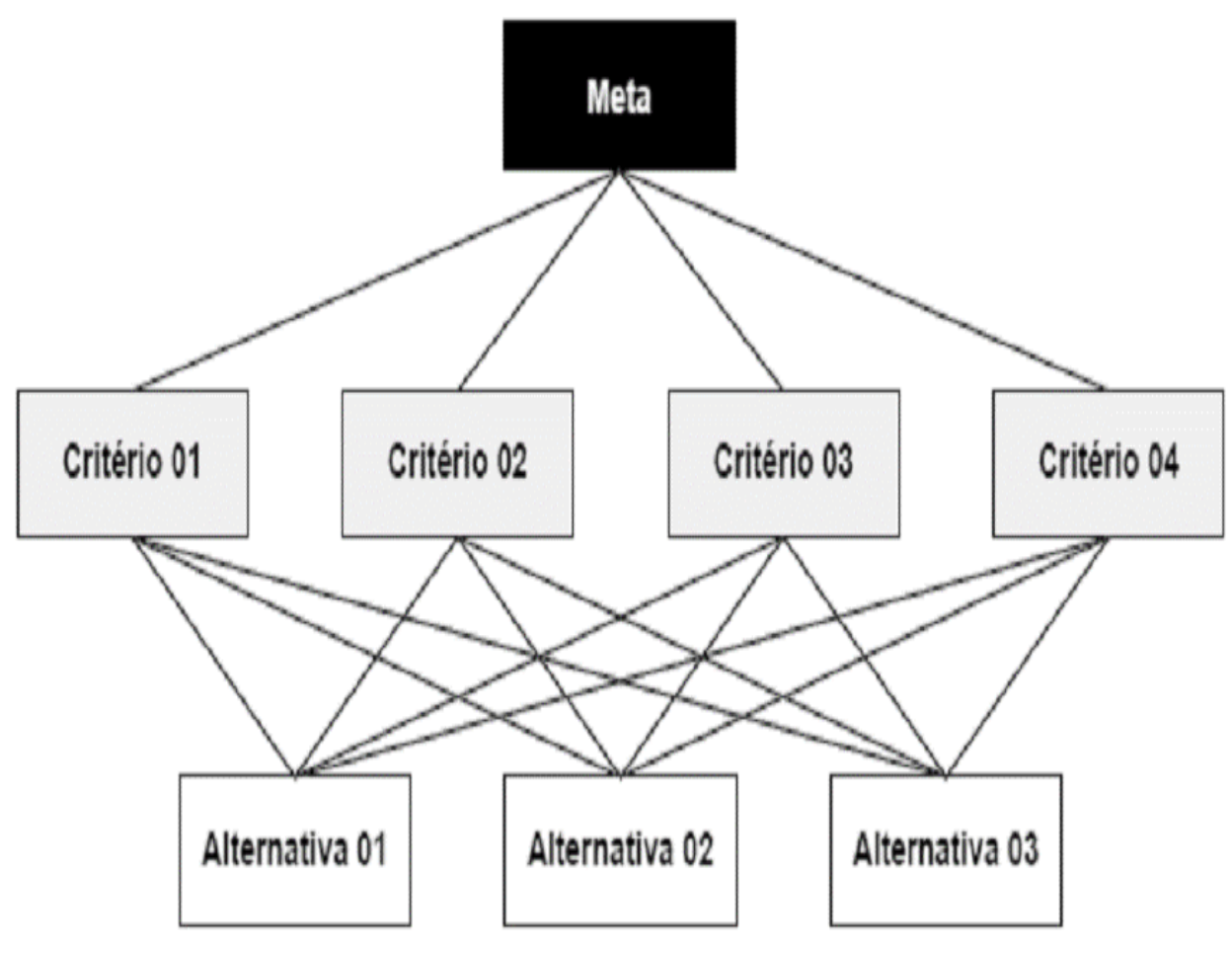

Fonte: Adaptado de Saaty (2005)

De acordo com Gomes et al. (2011), o modelo AHP é baseado na comparação par a par de uma série de critérios, de forma a evidenciar os critérios que apresentam maior relevância e qual a dimensão desta. Segundo Almeida (2013), essa comparação se baseia na avaliação centrada em uma escala semântica que abrange cinco níveis principais, de um total de nove níveis, sendo os demais valores intermediários, caso haja hesitação por parte do decisor. Abaixo segue a escala fundamental de Saaty na qual são baseadas todas as análises do método (Tabela 1).

Além disso, o método utiliza a escala de razão para efetuar todas as avaliações pertinentes e permite ainda a verificação da inconsistência nos julgamentos realizados pelos tomadores de decisão. Shimizu (2006) direciona algumas situações ideais para o uso dessa metodologia de análise multicritério, a saber: definição de prioridades; avaliação de custos e benefícios; alocação de recursos; mensuração de desempenho; avaliação ou pesquisa de mercado; especificação de requisitos; decisões 
estratégicas; planejamento e sequenciamento de atividades; previsão de cenários; negociação e resolução de conflitos, entre outros.

Tabela 1 - Escala Fundamental de Saaty

\begin{tabular}{ccc}
\hline Escala & Avaliação Numérica & Recíproco \\
\hline Extremamente preferido & 9 & $1 / 9$ \\
Muito forte a extremo & 8 & $1 / 8$ \\
Muito Fortemente preferido & 7 & $1 / 7$ \\
Forte a muito forte & 6 & $1 / 6$ \\
Fortemente preferido & 5 & $1 / 5$ \\
Moderado a forte & 4 & $1 / 4$ \\
Moderadamente preferido & 3 & $1 / 3$ \\
Igual a moderado & 2 & $1 / 2$ \\
Igualmente preferido & 1 & 1 \\
\hline
\end{tabular}

Fonte: Adaptado de Saaty (2005)

Dentre as principais vantagens associadas ao método, Vilas Boas (2006) relaciona os seguintes atributos:

- O AHP é um processo estruturado e pode facilmente ser documentado e repetido;

- Ideal na aplicação envolvendo julgamentos subjetivos;

- Utiliza tanto dados qualitativos quanto quantitativos;

- Admite medidas de consistência das preferências do decisor;

- Detém grande gama de aplicações documentadas na literatura;

- Uso adequado para grupos de decisão.

Apesar de ser um método bastante reconhecido e difundido por sua metodologia simples e por não requerer um processo de interpretação mais complexo para os decisores, existem muitos questionamentos quanto à efetividade de seu desempenho. Almeida (2013) destaca como principais problemas relativos a metodologia AHP:

a) Reversão de ordem;

b) Interpretação para os pesos dos critérios, indicando a importância relativa;

c) Uso da escala de razão para todas as ponderações, implicando na ocorrência do zero absoluto;

d) Interpretação numérica da escala verbal usada na elicitação.

Dessa forma, cabe ao analista ponderar os aspectos positivos e negativos do método, avaliar o tipo de problemática a ser avaliada e a eventual efetividade do modelo AHP, de forma a possibilitar um resultado coerente e que auxilie eficientemente o processo de tomada de decisão. 


\subsection{Fundamento matemático do AHP}

Após a especificação dos critérios e alternativas, ambos são submetidos à comparação par a par através da escala de razão (verbal) proposta por Saaty (1980), associando a cada resposta dada o seu respectivo valor na tabela 1. Após isso, determinaram-se as matrizes de comparação paritária, associando o nível de importância do relacionamento de cada fator $A_{i}$ com outro fator $A_{j}$. No caso do fator $A_{i}$ dominar o fator $A_{j}$, deve-se utilizar o valor referente ao recíproco na comparação entre $A_{j}$ e $A_{i}$. A equação 1 define a estrutura da matriz do modelo AHP.

$$
M=\left(\begin{array}{cccc}
1 & A_{12} & \cdots & A_{1} n \\
1 / A_{12} & 1 & \cdots & A_{2} n \\
\vdots & \vdots & \vdots & \vdots \\
1 / A_{1} n & 1 / A_{2} n & \cdots & 1
\end{array}\right)
$$

Depois de estipular as matrizes, o método recomenda a normalização $(M)$ destas, como forma de otimizar os dados. Essa normalização é realizada mediante 0 quociente entre o valor obtido pelo critério pelo somatório da coluna da matriz paritária inicial do referido critério (equação 2).

$$
N=\frac{A_{11}}{\sum\left(A_{11}+A_{21}+\cdots+A n_{1}\right)}
$$

A contribuição de cada critério na meta principal será determinada pela obtenção do vetor de Eigen ( $\lambda$ ) ou vetor prioridade. Esse vetor é determinado pela média aritmética dos valores normalizados em cada um dos critérios, devendo sempre obedecer a totalidade de $100 \%$ ou 1 . A equação 3 determina o vetor de Eigen:

$$
\lambda=\frac{\sum\left(A_{11}+A_{12}+\cdots+A_{1} n\right)}{n}
$$

A etapa seguinte da metodologia AHP consiste em verificar a inconsistência dos dados, ou seja, saber se os responsáveis pela tomada de decisão mantiveram uma coerência nas suas respostas. O índice de inconsistência baseia-se no vetor de Eigen, dessa forma, é preciso calcular o somatório do produto do valor correspondente ao vetor de Eigen para cada critério pelo total aferido por cada coluna da matriz de 
comparação paritária inicial, de forma a obter-se, segundo equação 4, o vetor principal de Eigen ( $\lambda$ máx).

$$
\lambda \text { máx }=\left(\lambda C 1 \times\left(\sum\left(\left(A_{11}+A_{21}+\cdots+A n_{1}\right)\right)\right)+\cdots+\left(\lambda C n \left(\sum \left(A_{1} n+A_{2} n+\cdots+\right.\right.\right.\right.
$$
Ann))

Com o vetor principal de Eigen obtido, cabe o cálculo do índice de consistência (Cl), que segundo Saaty (1980) é realizado através da equação 5:

$$
C I=\frac{\lambda m a ́ x-n}{n-1}
$$

Como forma de obter a adequação desse valor, Saaty (1980) ainda propõe o cálculo da taxa de consistência $(C R)$ que corresponde ao quociente entre o índice de consistência e o índice de consistência aleatória $(R I)$.

$$
C R=\frac{C I}{R I}
$$

Para que a matriz seja considerada consistente, ou seja, passível de confiabilidade, essa taxa não deve ultrapassar 0,1 ou $10 \%$. A tabela 2 apresenta os valores parametrizados do índice de consistência aleatória.

Tabela 2 - Índice de Consistência Aleatória $(R)$

\begin{tabular}{ccccccccccc}
$\mathbf{n}$ & $\mathbf{1}$ & $\mathbf{2}$ & $\mathbf{3}$ & $\mathbf{4}$ & $\mathbf{5}$ & $\mathbf{6}$ & $\mathbf{7}$ & $\mathbf{8}$ & $\mathbf{9}$ & $\mathbf{1 0}$ \\
\hline $\mathbf{R} \mathbf{r}$ & 0 & 0 & 0,58 & 0,9 & 1,12 & 1,24 & 1,32 & 1,41 & 1,45 & 1,49 \\
\hline
\end{tabular}

Fonte: Adaptado de Saaty (2005)

\section{CARACTERIZAÇÃO DA PESQUISA}

A seguinte pesquisa caracteriza-se como exploratório-descritiva, de caráter qualitativo e quantitativo. Em relação à tipologia de pesquisa, admite amostragem não probabilística intencional. Quanto aos procedimentos, a análise em foco foi estruturada a partir de um estudo de caso, que ponderou os principais aspectos e fundamentos ligados a um único objeto de estudo. 
Para o processo de obtenção de dados, optou-se pela utilização da pesquisa bibliográfica, aliada à pesquisa de campo. Utilizou-se ainda visitas in loco, aplicação de formulários estruturados e entrevistas informais realizadas com o gerente setorial.

$\mathrm{O}$ ambiente de pesquisa corresponde a uma empresa paraibana do segmento da construção civil com sede na cidade de Campina Grande - PB. Detém mais de 30 anos de experiência no mercado da construção, gerando 400 empregos diretos e mais de 1.000 indiretos, alocados nas áreas administrativas, de engenharia, financeira, comercial, de compras e segurança do trabalho. A empresa atua ainda nos estados de Pernambuco, Alagoas Rio Grande do Norte e Ceará.

\section{APLICAÇÃO DO MÉTODO AHP}

Primeiramente determinaram-se quais seriam os critérios a serem utilizados. Estes foram definidos com base nas entrevistas realizadas com o responsável pelo setor de Engenharia da construtora, destacando os principais pontos na condução das atividades operacionais. Os critérios avaliados serão os seguintes:

- Custo da atividade de projeto;

- Prazo de entrega da atividade;

- Disponibilidade de mão-de-obra;

- Disponibilidade de recursos materiais;

- Qualidade da atividade realizada;

- Riscos envolvidos na condução das atividades.

A segunda etapa consistiu em especificar as atividades de projetos que mereciam destaque na pesquisa. A empresa disponibilizou um sequenciamento de atividades que são inerentes à efetivação de uma obra de construção civil, mais especificamente, a construção de edificações e residências. Essas atividades foram categorizadas dentro de quatro grupos mais abrangentes: atividades de planejamento, atividades de execução de obra, atividades de instalações e atividades de acabamento. No quadro 1 é demonstrada esta classificação. 
Quadro 1 - Grupo de Atividades de Projeto executadas pela Empresa

\begin{tabular}{|c|c|c|c|}
\hline $\begin{array}{c}\text { PLANEJAMENTO } \\
\text { (A1) }\end{array}$ & $\begin{array}{c}\text { EXECUÇÃO DE } \\
\text { OBRA (A2) }\end{array}$ & $\begin{array}{l}\text { INSTALAÇÕES } \\
\text { (A3) }\end{array}$ & $\begin{array}{c}\text { ACABAMENTO } \\
\text { (A4) }\end{array}$ \\
\hline $\begin{array}{c}\text { Elaboração de } \\
\text { anteprojeto }\end{array}$ & Limpeza do terreno & Elétricas & $\begin{array}{c}\text { Acabamento/Revestimento } \\
\text { (pisos) }\end{array}$ \\
\hline Elaboração do projeto & Montagem do canteiro & Hidráulicas & $\begin{array}{c}\text { Esquadrias (portas e } \\
\text { janelas) }\end{array}$ \\
\hline $\begin{array}{l}\text { Aprovação legal do } \\
\text { projeto no órgão } \\
\text { responsável } \\
\text { (Prefeitura) }\end{array}$ & $\begin{array}{l}\text { Terraplanagem } \\
\text { (aterramento) }\end{array}$ & Telefonia & Forro e pintura \\
\hline Orçamento da obra & Locação da obra & Internet & Louça/Metais (pias, box) \\
\hline Planejamento da obra & $\begin{array}{c}\text { Fundação } \\
\text { (Infraestrutura) }\end{array}$ & - & Área externa/paisagismo \\
\hline- & $\begin{array}{c}\text { Estrutural (pilares, } \\
\text { vigas, lajes) }\end{array}$ & - & Limpeza final \\
\hline- & Paredes e vedações & - & - \\
\hline- & Cobertura (telhado) & - & - \\
\hline
\end{tabular}

Após isso, efetivou-se a hierarquização dos critérios e grupos de atividades (alternativas), atendendo a metodologia AHP. A figura 2 apresenta a hierarquia dos critérios e atividades a serem avaliados.

Figura 2 - Hierarquia de Critérios/Atividades

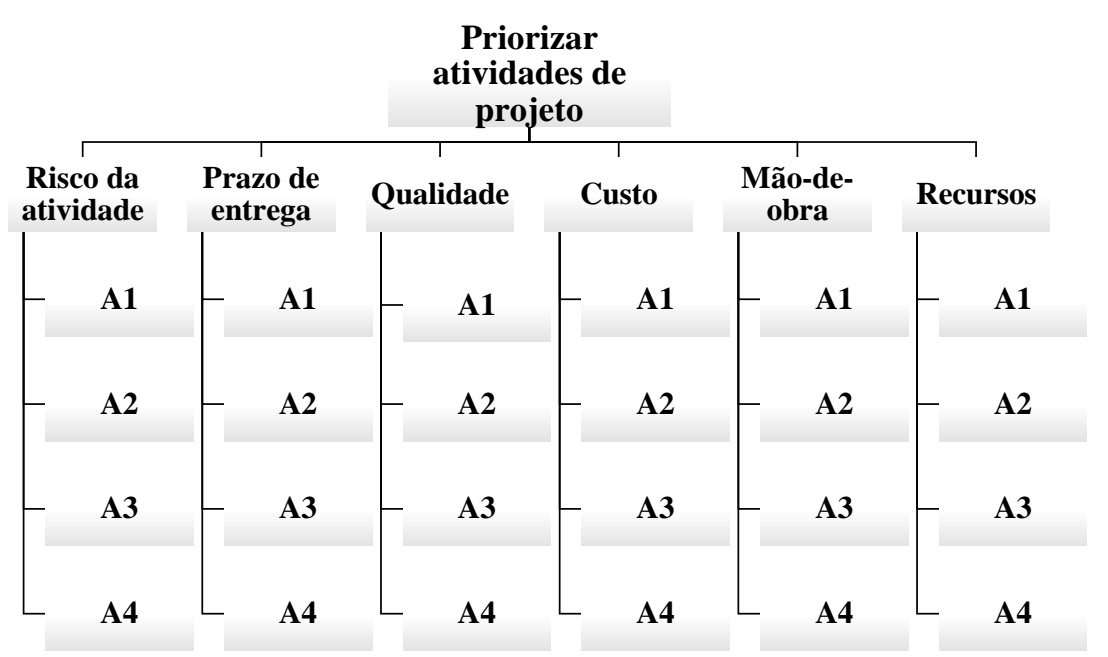

Realizou-se as comparações par a par entre os critérios e depois a normalização dos resultados obtidos de acordo com o apresentado nas tabelas 3 e 4 . 
Tabela 3 - Matriz de Comparação par a par

\begin{tabular}{ccccccc}
\hline & Risco & Prazo & Qualidade & Custo & MO & Recursos \\
\hline Risco & 1,00 & 0,33 & 0,20 & 0,14 & 1,00 & 1,00 \\
Prazo & 3,00 & 1,00 & 0,20 & 0,14 & 1,00 & 1,00 \\
Qualidade & 5,00 & 5,00 & 1,00 & 0,33 & 5,00 & 3,00 \\
Custo & 7,00 & 7,00 & 3,00 & 1,00 & 5,00 & 7,00 \\
Mo & 1,00 & 1,00 & 0,20 & 0,20 & 1,00 & 1,00 \\
Recursos & 1,00 & 1,00 & 0,33 & 0,14 & 1,00 & 1,00 \\
\hline
\end{tabular}

Tabela 4 - Matriz de Comparação par a par Normalizada

\begin{tabular}{ccccccc}
\hline & Risco & Prazo & Qualidade & Custo & MO & Recursos \\
\hline Risco & 0,055556 & 0,021526 & 0,040568 & 0,071795 & 0,071429 & 0,071429 \\
Prazo & 0,166667 & 0,065232 & 0,040568 & 0,071795 & 0,071429 & 0,071429 \\
Qualidade & 0,277778 & 0,326158 & 0,202840 & 0,169231 & 0,357143 & 0,214286 \\
Custo & 0,388889 & 0,456621 & 0,608519 & 0,512821 & 0,357143 & 0,500000 \\
Mo & 0,055556 & 0,065232 & 0,040568 & 0,102564 & 0,071429 & 0,071429 \\
Recursos & 0,055556 & 0,065232 & 0,066937 & 0,071795 & 0,071429 & 0,071429 \\
\hline
\end{tabular}

A partir da normalização da matriz foi possível calcular o vetor de Eigen $(\lambda)$ para cada um dos critérios em análise e em seguida obter um valor para o vetor principal de Eigen ( $\lambda$ máx). O vetor de Eigen irá indicar o peso de cada critério, conforme tabela 5.

Tabela 5 - Resultados do Cálculo do Vetor de Eigen e do Vetor Principal de Eigen

$\begin{array}{ccc}\text { Cálculo do Vetor de Eigen }(\lambda) & \% \\ \text { Risco } & 0,055384 & 5,538366 \\ \text { Prazo } & 0,081186 & 8,118637 \\ \text { Qualidade } & 0,257906 & 25,79058 \\ \text { Custo } & 0,470665 & 47,06654 \\ \text { MO } & 0,067796 & 6,779605 \\ \text { Recursos } & 0,067063 & 6,706271\end{array}$

Vetor principal de Eigen ( $\lambda$ máx)

6,31878865

Foram calculados o índice de consistência $(C l=0,0637577)$ e a taxa de consistência da matriz $(C R=0,0514175)$. Como o índice $C R$ indicou um valor de $5,14 \%$, pode-se inferir que a matriz é consistente e que os dados obtidos admitem confiabilidade. 


\section{RESULTADOS E DISCUSSÕES}

A partir dos resultados da aplicação do AHP, o critério custo mostrou-se fundamental para a priorização das atividades, já o critério qualidade assume o segundo lugar em relevância. O foco nesses dois aspectos é justificável pelo fato de ambos serem pilares básicos nos quais a empresa atua.

O custo corresponde a um ponto determinante na condução dos negócios da empresa, onde a redução destes implica em novos investimentos e melhores projetos, assim como na aferição de lucros mais significativos. A qualidade consiste em fator relevante na conclusão das obras, bem como está associada a uma exigência do mercado e do próprio cliente, que busca informações sobre as construtoras mais confiáveis em relação a esse critério.

Os demais critérios apresentaram um desempenho aquém do esperado em se tratando de uma empresa do segmento construção civil, principalmente o fator prazo, uma vez que os prazos determinam a competitividade no mercado da construção. Dessa forma, negligenciar tal aspecto pode consistir em uma estratégia de negócios perigosa, originando uma redução na carteira de clientes e a perda da credibilidade perante o mercado.

Os fatores risco da atividade, mão-de-obra e recursos materiais, estão de certa forma associados, porém deveriam deter uma maior atenção pela empresa, já que o uso de recursos de qualidade e de um capital humano bem treinado pode não só reduzir os riscos das atividades e os custos no longo prazo, como também garantir um produto final com maior qualidade.

Efetivou-se ainda uma análise de cada grupo de atividade (alternativas) em relação a cada um dos critérios. Dessa forma, foi possível determinar a influência de cada grupo de atividade em detrimento a cada critério e estabelecer qual destes terá maior prioridade no processo decisório (figura 3). 
Figura 3 - Percentual de Relevância de cada Atividade em relação aos Critérios

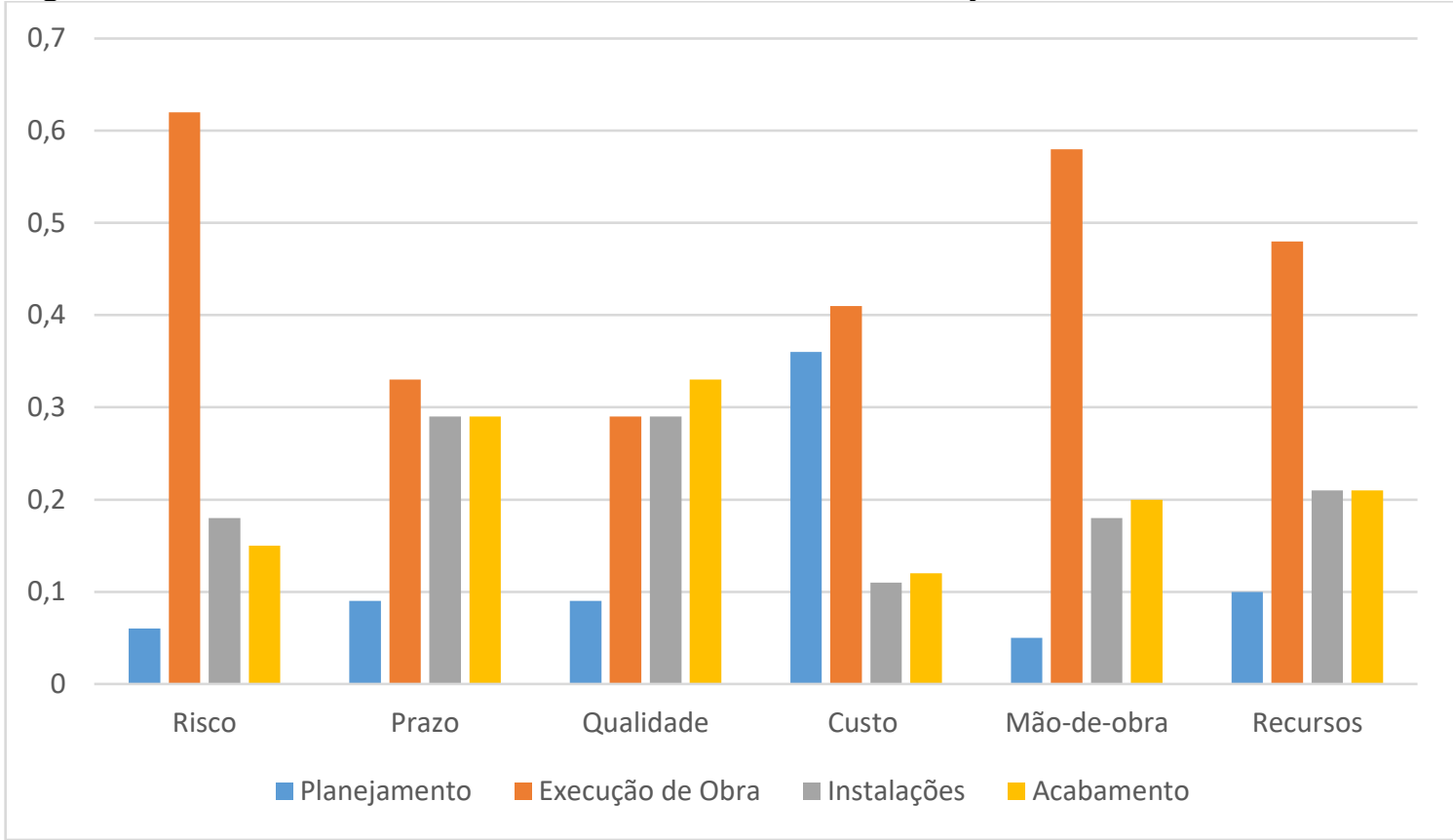

A partir disso, verificou-se que o grupo de atividades execução de obra tem importância fundamental nas decisões que envolvem os projetos de construção, tendo em vista seu desempenho satisfatório em relação a todos os critérios de avaliação. Os grupos de atividades de instalação e de acabamento encontram-se em um patamar intermediário em todos os critérios. São atividades de menor relevância se comparadas com as de execução de obra, porém essenciais à finalização dos projetos. Seu resultado inferior é justificado até mesmo pelo fato de sua efetivação depender da finalização da etapa de execução de obra.

Um ponto de destaque se refere ao desempenho baixo do grupo de atividades de planejamento em grande parte dos critérios de decisão. Apenas no critério custo, há uma significativa participação do planejamento, porém em critérios como qualidade e prazo que deveriam admitir uma maior consistência dessas atividades de planejamento, esse aspecto é negligenciado.

Buscou-se ainda determinar a relevância de cada grupo de atividade de projeto sob a perspectiva global. Para tanto, obteve-se o produto entre os pesos de cada critério pelos valores correspondentes a participação de cada atividade. A tabela 6 indica os percentuais de prioridade de cada grupo de atividade em relação aos critérios e a porcentagem de prioridade final. 
Tabela 6 - Percentuais de prioridade

\begin{tabular}{|c|c|c|c|c|c|c|c|}
\hline & Risco & Prazo & Qualidade & Custo & $\begin{array}{l}\text { Mão-de- } \\
\text { obra }\end{array}$ & Recursos & $\begin{array}{l}\text { Prioridade } \\
\text { Final (\%) }\end{array}$ \\
\hline Planejamento & 0,06 & 0,09 & 0,09 & 0,36 & 0,05 & 0,1 & 21,33769392 \\
\hline $\begin{array}{c}\text { Execução de } \\
\text { Obra }\end{array}$ & 0,62 & 0,33 & 0,29 & 0,41 & 0,58 & 0,48 & 40,04066827 \\
\hline Instalações & 0,18 & 0,29 & 0,29 & 0,11 & 0,18 & 0,21 & 18,6365439 \\
\hline Acabamento & 0,15 & 0,29 & 0,33 & 0,12 & 0,2 & 0,21 & 20,10827362 \\
\hline
\end{tabular}

Pode-se inferir que o grupo de atividades execução de obra tem uma relevância efetiva no que concerne à priorização de atividades de projeto, por se tratar de um grupo de atividades complexas e determinantes em um projeto de construção civil, exigindo maiores prazos de entrega, mão-de-obra mais qualificada, além de recursos financeiros e insumos elevados para sua efetivação.

Apesar do grupo de atividades de planejamento admitir um desempenho notadamente mais baixo na comparação com o grupo de atividades de execução de obra quando confrontada isoladamente em relação aos critérios, na avaliação final acaba por otimizar sua participação global, uma vez que estas atividades alcançaram um melhor desempenho em relação ao critério custo que possui grande relevância na decisão final. A figura 4 sintetiza a prioridade final dos grupos de atividades de projeto.

Figura 4 - Prioridade Final de cada Grupo de Atividade

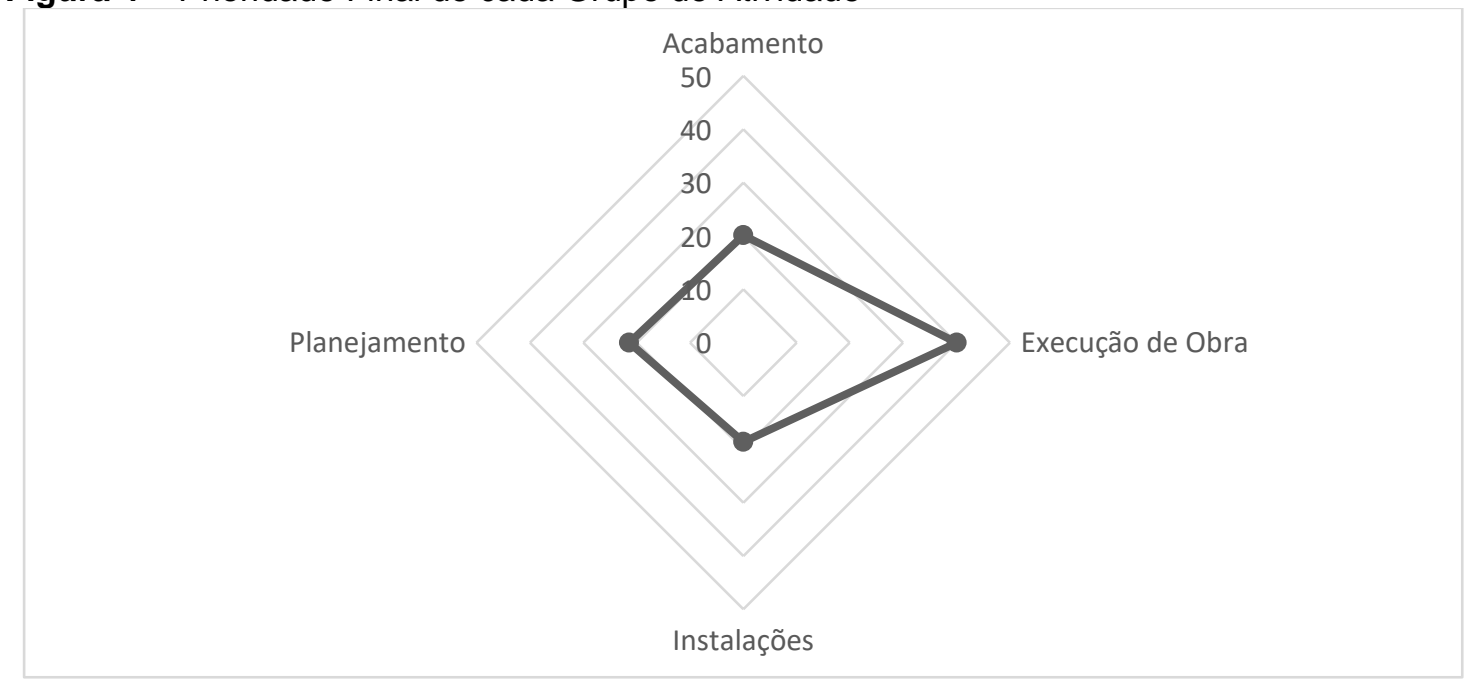

Observa-se ainda que o desempenho das demais atividades em relação à meta principal admite um percentual semelhante com pouca variação, dessa forma, há uma uniformidade na relevância dessas atividades em detrimento a priorização das atividades de projetos. Sendo assim, cabe a empresa, utilizar de forma eficiente os 
resultados obtidos de modo a adequar seu planejamento e gerenciamento de projetos as suas necessidades principais.

\section{CONSIDERAÇÕES FINAIS}

No que concerne à priorização das atividades de projeto, foi possível verificar que o critério custo assume grande importância, ou seja, muito embora a empresa atue com base em pressupostos como qualidade e produtividade, o custo ainda é fator significativo na condução do planejamento de projetos e tem máxima importância na determinação de quais atividades consumirão maior parte do orçamento.

Em relação aos grupos de atividades de projeto, identificou-se que o grupo execução de obra assumiu maior relevância na decisão de priorização de atividades de projetos, tendo em vista ser composta por subatividades essenciais a construção civil, demandando maiores prazos, mão-de-obra, recursos financeiros e insumos para sua execução.

O estudo sugere para trabalhos futuros, a ampliação do uso do método AHP nas subatividades pertencentes aos grupos de atividades relacionados, como forma de viabilizar um melhor apoio no que se refere à priorização das atividades no processo de decisão. Além disso, seria possível viabilizar a priorização das atividades de projeto mediante o uso de outras metodologias multicritério.

Dessa forma, a associação de métodos multicritérios visando a determinação de atividades prioritárias de projetos surge como uma abordagem efetiva de apoio a decisão, ao planejamento e a gestão de projetos no âmbito da construção civil. O método AHP mostrou-se eficiente para a finalidade empregada, possibilitando uma análise mais simples para os tomadores de decisão, facilitando as avaliações e novas aplicações do método para outras finalidades.

\section{REFERÊNCIAS}

ALENCAR, L. H.; ALMEIDA, A. T.; MOTA, C. M. M. Sistemática proposta para seleção de fornecedores em gestão de projetos. Gest. Prod., São Carlos, v. 14, n. 3, p. 477-487, set./dez. 2007. https://doi.org/10.1590/S0104-530X2007000300005

ALMEIDA, A T. Processo de decisão nas organizações: construindo modelos de decisão multicritério. São Paulo: Atlas, 2013. 
ARAÚJO, M. C. B.; ALENCAR, L. H.; REIS, A. C.; MOTA, C. M. M. Modelo multicritério para a seleção de contratados em projetos de construção civil. In: SIMPÓSIO BRASILEIRO DE PESQUISA OPERACIONAL, 42., 2015. [Anais...] Porto de Galinhas, Pernambuco, 2015.

AZEVEDO, R. C.; LACERDA, R. T. O.; ENSSLIN, L.; JUNGLES, A. E.; ENSSLIN, S. R. Performance Measurement to Aid Decision Making in the Budgeting Process for ApartmentBuilding Construction: Case Study Using MCDA-C. Journal of Construction Engineering and Management, v. 139, p. 225-235, 2013. https://doi.org/10.1061/(ASCE)CO.1943$\underline{7862.0000587}$

BANA e COSTA, C. Introdução geral a abordagens multicritério de apoio à tomada de decisão. Investigação Operacional, v.8, n.1, p 117-139, 1988.

BEN, F. Utilização do método AHP em decisões de investimento ambiental. In: ENCONTRO NACIONAL DE ENGENHARIA DE PRODUÇÃO, 26., 2006. [Anais...]. Fortaleza, 2006.

BORNIA, A. C.; WERNKE, R. A contabilidade gerencial e os métodos multicriteriais. Revista Contabilidade \& Finanças, v. 14, n. 25, p. 60 - 71, jan./abr., 2001.

https://doi.org/10.1590/S1519-70772001000100004

CAVALCANTE, C. A. V.; ALMEIDA, A. T. Modelo multicritério de apoio a decisão para o planejamento de manutenção preventiva utilizando PROMETHEE II em situações de incerteza. Pesquisa Operacional, v. 25, n.2, p.279-296, 2005. https://doi.org/10.1590/S010174382005000200007

COSTA, J. F. S.; RODRIGUES, M. M.; FELIPE, A. P. M. Utilização do método de análise hierárquica (AHP) para escolha da interface telefônica. In: ENCONTRO NACIONAL DE ENGENHARIA DE PRODUÇÃO, 38., 2008. [Anais...]

FAMÁ, C. C. G.; ALENCAR, L. H.; ALMEIDA, A. T. Assignment of Critical Path for a Building Site Based on Multicriteria Approach. In: Industrial Engineering and Engineering Management (IEEM), 2015. https://doi.org/10.1109/IEEM.2015.7385859

GOMES, L. F. A. M.; MOREIRA, A. M. M. Da informação à tomada de decisão: agregando valor através dos métodos multicritério. RECITEC, Recife, v. 2, n. 2, p. 117-139, 1998.

GOMES, L. F. A. M; GOMES, C. F. S; ALMEIDA, A. T. Tomada de decisão gerencial: enfoque multicritério. São Paulo: Atlas, 2002.

GOMES, L. F. A. M. Tomada de decisões em cenários complexos. São Paulo: Pioneira Thompson Learning, 2004.

GOMES, L. F. A. M; ARAYA, M. C. G.; CARIGNANO, C. Tomada de Decisões em Cenários Complexos: introdução aos métodos discretos do apoio multicritério à decisão. Cengage Learning: São Paulo, 2011.

LIMA, J. P.; RAMOS, R. A. R.; FERNANDES JÚNIOR, J. L. Priorização de pavimentos com o uso da metodologia de análise multicritério e SIG. ENGEVISTA, v. 10, n. 2, p. 132-144; Dezembro, 2008. https://doi.org/10.22409/engevista.v10i2.220

MOTA, C. M. M.; ALENCAR, L. H.; ALMEIDA, A. T. A multiple criteria decision model for assigning priorities to activities in project management. International Journal of Project Management, v. 27, p. 175-181, 2009. https://doi.org/10.1016/j.ijproman.2008.08.005 
MOTA, C. M. M.; ALMEIDA, A. T. Método multicritério ELECTRE IV-H para priorização de atividades em projetos. Pesqui. Oper. [online], v. 27, n.2, p. 247-269, 2007.

https://doi.org/10.1590/S0101-74382007000200004

ROY, B. Multicriteria methodology for decision aiding. Kluwer Academic Publishers, 1996. https://doi.org/10.1007/978-1-4757-2500-1

SAATY, T L. The Analytic Hierarchy Process. New York: McGraw - Hill, 1980. https://doi.org/10.21236/ADA214804

SAATY, T L. Theory and applications of the analytic network process: decision making with benefits, opportunities, costs and risks. Pittsburgh: RWS Publications, 2005.

SCHMOLDT, D. L.; PETERSON, D. L. Using de AHP in a workshop setting to elicit and prioritize fire research needs. E.U.A.: Forest Science, 1997.

SHIMIZU, T. Decisão nas organizações. 2. ed. São Paulo: Atlas, 2006.

SILVA, M. C. G.; SILVA, N. P.; JUSTUS, C. C.; COLMENERO, J. C. Metodologia para identificação de potenciais locais para a implantação de usina de reciclagem de resíduos da construção civil. Espacios, v. 34, n. 4, p. 2, 2013.

SINDICATO NACIONAL DA CONSTRUÇÃO PESADA - INFRAESTRUTURA (SINICON). Disponível em: http://www.sinicon.org.br. Acesso em: 13 dez. 2017.

SONDAGEM INDÚSTRIA DA CONSTRUÇÃO. Informativo da Confederação Nacional da Indústria. Ano 8, n. 9, set. 2017. https://doi.org/10.22409/tn.9i12.p6148

SZAJUBOK, N. K.; ALENCAR, L. H.; ALMEIDA, A. T. Modelo de gerenciamento de materiais na construção civil utilizando avaliação multicritério. Produção, v. 16, n. 2, p. 303-318, Maio/Ago. 2006. https://doi.org/10.1590/S0103-65132006000200010

SZAJUBOK, N. K.; MOTA, C. M. M.; ALMEIDA, A. T. Uso do método multicritério ELECTRE TRI para classificação de estoques na construção civil. Pesquisa Operacional, v.26, n.3, p.625-648, set./ dez. 2006. https://doi.org/10.1590/S0101-74382006000300010

TOMA, T.; ASHARIF, M. R. AHP coefficients optimization technique based on GA. Japão: Department of Information Engineering of University of Ryukyus, 2003.

VILAS BOAS, C. L. de. Modelo multicritérios de apoio a decisão aplicado ao uso múltiplo de reservatórios: estudo da barragem do Ribeirão João Leite. Dissertação (Mestrado em Gestão Econômica do Meio Ambiente). Universidade de Brasília - UNB. Departamento de Economia. Brasília, 2006.

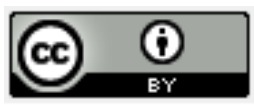

Artigo recebido em: 17/05/2018 e aceito para publicação em: 01/12/2019 DOI: http://dx.doi.org/10.14488/1676-1901.v19i4.3255 
